\title{
Pushing Towards Room-Temperature Superconductivity
}

\author{
Two independent studies report superconductivity at record high temperatures in \\ hydrogen-rich materials under extreme pressure.
}

\section{by Eva Zurek*}

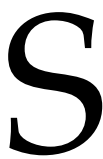

uperconductivity, the ability of a material to conduct electricity without any resistance, was first observed in 1911 in solid mercury below a critical temperature $\left(T_{c}\right)$ of $4.2 \mathrm{~K}$. Ever since, countless scientists have been searching for a material whose $T_{c}$ exceeds room temperature. For a long time this holy grail seemed unattainable-a linear extrapolation of research progress from 1911 to 1970 suggested that $T_{C}$ would reach room temperature around the year 2840! The discovery of high-temperature superconductivity in copper oxides raised $T_{c}$ above liquid helium temperature. Since 1994, one of the copper oxides has held the record for the highest $T_{\mathcal{C}}(133 \mathrm{~K}$ at atmospheric pressure and $164 \mathrm{~K}$ under high pressure). Despite intense research, it took another 20 years to beat this record in a completely new class of systems: In 2015, the compression of hydrogen sulfide to $150 \mathrm{GPa}$, or about $40 \%$ of the pressure found in Earth's core, yielded a $T_{\mathcal{C}}$ of $203 \mathrm{~K}$ [1]. Remarkably, two independent
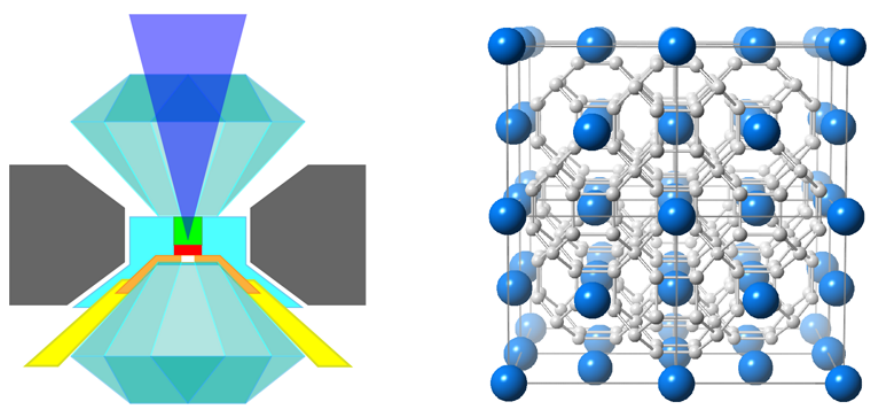

Figure 1: (Left) Scheme of the diamond anvil cell used to study the behavior of $\mathrm{LaH}_{10}$ under high static pressure. (Right) Sketch of the cage-like crystal structure $\left(\mathrm{LaH}_{10}\right)$ thought to be responsible for the high-temperature superconductivity observed by the groups of Hemley [2] and Eremets [3]. ((Left) APS/Alan Stonebraker; (Right) E. Zurek, adapted by APS/Alan Stonebraker)

\footnotetext{
*Department of Chemistry, University at Buffalo, SUNY, Buffalo NY, USA
}

groups, the first led by Russell Hemley at the George Washington University in Washington, DC [2], and the second by Mikhail Eremets at the Max Planck Institute for Chemistry, Germany [3], have now reported experiments indicating that a hydride of lanthanum compressed to $170-185 \mathrm{GPa}$ has a $T_{c}$ of $250-260 \mathrm{~K}[2,3]$. The results bode well for the search for room-temperature superconductors-the reported materials could already work without the need for cooling on an average winter night in the Arctic!

In 1968, physicist Neil Ashcroft predicted that metallic hydrogen should have all of the properties required to be a high-temperature superconductor according to BardeenCooper-Schrieffer (BCS) theory [4]. Unfortunately, metallizing hydrogen in static compression experiments turned out to be extremely difficult. Ashcroft further predicted that certain hydrogen-rich solids might become metallic at lower pressures than elemental hydrogen and that they would possess the same properties conducive to high-temperature superconductivity [5]. This hypothesis catalyzed the search for superconductivity in compressed hydrides-solids containing hydrogen atoms bonded to other elements.

To study superconductivity in these compressed materials, researchers need to carry out static high-pressure experiments in which the materials are squeezed in diamond anvil cells (Fig. 1, left). Such experiments are expensive, technically challenging, and can be difficult to interpret. What's more, the material phases that are stable under pressure can be different than the ones we know to occur at atmospheric conditions. As a result, quantum-mechanics-based computations have become extremely important in guiding these experiments, in particular by pinpointing promising compounds [6].

In the last decade, these theoretical and computational techniques have focused on binary hydrides. Researchers have calculated extremely high $T_{c}$ values - some even surpassing room temperature-for hydrides containing alkaline-earth metals or rare-earth metals [7]. In 2017, for example, groups led by Hemley [8] and Yanming Ma [9] predicted that certain rare-earth hydrides with a large hydrogen-to-metal ratio would become stable at pressures reachable in diamond anvil cells. These hydrogen-rich materials have crystal structures that are reminiscent of the cage- 
like structures of chemical compounds called clathrates. One of the most promising hydrides, lanthanum hydride $\left(\mathrm{LaH}_{10}\right)$, consists of a hydrogen lattice made of polyhedra with square or hexagonal faces (Fig. 1, right), with a rareearth metal atom lying at the center of each polyhedron. Assuming that this system could be described by BCS theory, the researchers predicted its $T_{\mathcal{C}}$ to lie between 270 and $290 \mathrm{~K}$ at $\sim 200 \mathrm{GPa}[8,9]$.

In early 2018, Hemley's group managed to synthesize $\mathrm{LaH}_{10}$ [10]. Now, Hemley's [2] and Eremets' [3] teams have reported experimental signatures of superconductivity in $\mathrm{LaH}_{10}$ under the extreme pressures obtained inside diamond anvil cells. In order to accurately measure the electrical resistance within their micrometer-wide $\mathrm{LaH}_{10}$ samples, both groups had to ensure reliable contacts between sample and electrodes and to control the sample compositions and conditions (for instance, by preventing the formation of additional phases). Hemley's group developed a novel synthesis technique in which $\mathrm{LaH}_{10}$ was produced in situ using ammonia borane $\left(\mathrm{NH}_{3} \mathrm{BH}_{3}\right)$ as the hydrogen source. As the sample was cooled under a 185-GPa pressure, they observed a dramatic resistance drop that indicated a $T_{c}$ of $260 \mathrm{~K}$. A second set of experiments suggested that $T_{c}$ could become as high as $280 \mathrm{~K}$ at $200 \mathrm{GPa}$. X-ray diffraction measurements suggested that the superconducting phase could be $\mathrm{LaH}_{10}$ and ruled out the possibility that the resistance drop was caused by a temperature-induced structural transition.

Eremets' team synthesized the hydride via a direct reaction of elemental lanthanum or lanthanum trihydride $\left(\mathrm{LaH}_{3}\right)$ and hydrogen. They observed sharp drops in the resistance, from which they derived $T_{\mathcal{C}}$ as a function of pressure. For $\mathrm{LaH}_{10}$, the highest $T_{\mathcal{C}}$ value of $\sim 250 \mathrm{~K}$ was measured at $\sim 170 \mathrm{GPa}$. Another unidentified hydride phase, which coexisted with $\mathrm{LaH}_{10}$ in the same sample, exhibited a $T_{c}$ of $215 \mathrm{~K}$ [11]. Eremets' team also observed two characteristic signatures of superconductivity. First, the application of a magnetic field reduced $T_{c}$, as expected for a type-II BCS superconductor. Second, $T_{c}$ exhibited the so-called isotope effect-it decreased when hydrogen was replaced by heavier deuterium [12].

These data are strongly suggestive of superconductivity, but to prove it beyond a shadow of a doubt, it would be necessary to observe the Meissner effect-the expulsion of a magnetic field from a material when it becomes superconducting. Measuring this effect is, however, challenging: for the previous high- $T_{\mathcal{C}}$ record holder, sulfur hydride, the Meissner effect could only be demonstrated several years after the initial report of superconductivity. Since the lanthanum hydride samples are significantly smaller than the sulfur hydride samples, demonstrating the Meissner effect for $\mathrm{LaH}_{10}$ will require substantial experimental efforts.

Further theoretical and experimental work will also be needed to identify the multiple crystalline lattices contained in the samples. The data strongly suggest that one of these is $\mathrm{LaH}_{10}$, but the identity of the other structures remains unknown. This information will be essential to understand the relationship between crystal structure and superconductivity and, possibly, to unveil new superconducting phases that might have an even higher $T_{\mathcal{c}}$. And the high $T_{\mathcal{c}}$ of $\mathrm{LaH}_{10}$ will certainly motivate experimentalists to investigate similar systems, such as yttrium hydride, whose predicted $T_{c}$ exceeds room temperature $[8,9]$.

In the field of superconductivity, most breakthroughs have been unexpected, often defying the conventional wisdom of the time. The hydride case shows that this may no longer be the case: theoretical calculations can be used to rationally design new materials that are high- $T_{c}$, BCS-type superconductors. Computational advances will make it possible to pinpoint other complex species that, unlike binary hydrides such as $\mathrm{LaH}_{10}$, may remain stable when decompressed. And new techniques for crystal synthesis and characterization will allow the experimental test of theoretical ideas. Thanks to this feedback loop between theory and experiment, we might soon have a superconductor that works close to room temperature and at pressures that could be achieved in much simpler devices than diamond anvil cells, such as the presses used to compress powders into pharmaceutical tablets.

This research is published in Physical Review Letters.

\section{REFERENCES}

[1] A. P. Drozdov, M. I. Eremets, I. A. Troyan, V. Ksenofontov, and S. I. Shylin, "Conventional superconductivity at 203 kelvin at high pressures in the sulfur hydride system," Nature 525, 73 (2015).

[2] M. Somayazulu, M. Ahart, A. K. Mishra, Z. M. Geballe, M. Baldini, Y. Meng, V. V. Struzhkin, and R. J. Hemley, "Evidence for superconductivity above $260 \mathrm{~K}$ in lanthanum superhydride at megabar pressures," Phys. Rev. Lett. 122, 027001 (2019).

[3] A. P. Drozdov et al., "Superconductivity at $250 \mathrm{~K}$ in lanthanum hydride under high pressures," arXiv:1812.01561.

[4] N. W. Ashcroft, "Metallic hydrogen: A high-temperature superconductor?" Phys. Rev. Lett. 21, 1748 (1968).

[5] N. W. Ashcroft, "Hydrogen dominant metallic alloys: High temperature superconductors?" Phys. Rev. Lett. 92, 187002 (2004).

[6] E. Zurek and W. Grochala, "Predicting crystal structures and properties of matter under extreme conditions via quantum mechanics: The pressure is on," Phys. Chem. Chem. Phys. 17, 2917 (2015).

[7] E. Zurek and $\mathrm{T}$. Bi, "High-temperature superconductivity in alkaline and rare earth polyhydrides at high pressure: A theoretical perspective," J. Chem. Phys. (to be published).

[8] H. Liu, I. I. Naumov, R. Hoffmann, N. W. Ashcroft, and R. J. Hemley, "Potential high- $T_{c}$ superconducting lanthanum and yttrium hydrides at high pressure," Proc. Natl. Acad. Sci. U.S.A. 114, 6990 (2017).

[9] F. Peng, Y. Sun, C. J. Pickard, R. J. Needs, Q. Wu, and Y. Ma, "Hydrogen clathrate structures in rare earth hydrides at high pressures: Possible route to room-temperature superconductivity," Phys. Rev. Lett. 119, 107001 (2017). 
[10] Z. M. Geballe, H. Liu, A. K. Mishra, M. Ahart, M. Somayazulu, Y. Meng, M. Baldini, and R. J. Hemley, "Synthesis and stability of lanthanum superhydrides," Angew. Chem. Int. Ed. 57, 688 (2018).

[11] A. P. Drozdov, V. S. Minkov, S. P. Besedin, P. P. Kong, M. A. Kuzovnikov, D. A. Knyazev, and M. I. Eremets, "Superconductivity at $215 \mathrm{~K}$ in lanthanum hydride at high pressures," arXiv:1808.07039.
[12] While this observation is in line with BCS theory, it is important to note that the $T_{C}$ comparison needed to establish the isotope effect assumes that the superconducting deuteride and hydride of lanthanum have the same structure-an assumption that could not be tested in these experiments.

10.1103/Physics.12.1 